\title{
Le rôle des promotions foncières et immobilières dans la production des périphéries : application à la Belgique et à ses nouveaux espaces résidentiels
}

The role of real estate developers for the production of peripheries: Application for Belgium and its new residential quarters

Die Rolle der Eigentumsverhältnisse und der Aktivität von

Wohnbaugesellschaften für die Entwicklung der städtischen Außenviertel : Der

Fall der neuen Wohnviertel in Belgien

Jean-Marie Halleux

\section{CpenEdition}

\section{Édition électronique}

URL : http://journals.openedition.org/rge/285

DOI : $10.4000 /$ rge. 285

ISSN : 2108-6478

\section{Éditeur}

Association des géographes de l'Est

\section{Édition imprimée}

Date de publication : 1 juin 2005

Pagination : 161-173

ISSN : 0035-3213

\section{Référence électronique}

Jean-Marie Halleux, « Le rôle des promotions foncières et immobilières dans la production des périphéries : application à la Belgique et à ses nouveaux espaces résidentiels », Revue Géographique de l'Est [En ligne], vol. 45 / 3-4 | 2005, mis en ligne le 18 mai 2009, consulté le 08 septembre 2020. URL: http://journals.openedition.org/rge/285; DOI : https://doi.org/10.4000/rge.285 


\section{Le rôle des promotions foncières et immobilières dans la production des périphéries : application à la Belgique et à ses nouveaux espaces résidentiels}

The role of real estate developers for the production of peripheries: Application for Belgium and its new residential quarters

Die Rolle der Eigentumsverhältnisse und der Aktivität von

Wohnbaugesellschaften für die Entwicklung der städtischen Außenviertel : Der

Fall der neuen Wohnviertel in Belgien

Jean-Marie Halleux

\section{NOTE DE L'ÉDITEUR}

Article reçu le 22 août 2005, accepté le 5 décembre 2005

\section{Introduction}

1 Le développement des périphéries résidentielles est en Belgique un phénomène ayant suscité de nombreuses recherches, consacrées tant aux dimensions spatiales, sociales et démographiques ${ }^{1}$. Il est vrai qu'en raison du caractère particulièrement soutenu et éclaté de la périurbanisation qui caractérise le pays (J.-M. Halleux et al., 2002), les chercheurs belges se devaient de s'intéresser à cette problématique. Dans le cadre de cet article, nous viserons à prendre en compte une dimension du phénomène n'ayant encore été que peu analysée, celle du rôle des promotions foncières et immobilières. 
Malgré l'importance des enjeux spatiaux et économiques qu'ils représentent, les secteurs économiques de la promotion foncière et immobilière demeurent mal connus. Certes, il s'agit de mondes difficiles d'accès, complexes, enchevêtrés, où les relations se nouent et se défont entre des acteurs multiples. Mais cette complexité n'explique pas tout et force est de reconnaître que le rôle des promoteurs dans la production des espaces urbains continue à être négligé par les chercheurs actifs en sciences régionales et territoriales.

2 Par promotion immobilière, nous entendons une opération ayant pour objet la création ou la rénovation d'un produit immobilier bâti. Préalablement à la réalisation d'une promotion immobilière, il peut être nécessaire de réaliser une promotion foncière, consistant ici en la production d'une parcelle de terrain directement disponible pour la construction. Le promoteur d'une opération foncière ou immobilière est l'acteur qui prend l'initiative de sa réalisation. Egalement appelé maître d'ouvrage, le promoteur est en relation avec les maîtres d'œuvre et les entreprises qu'il choisit. Il s'assure de la faisabilité de l'opération, en définissant sa localisation, ses objectifs et en déterminant son plan de financement. Les promoteurs peuvent être animés par deux objectifs distincts: soit l'auto-occupation, soit la commercialisation. A l'objectif de l'autooccupation correspond la filière de "l'auto-promotion", où le futur occupant de l'immeuble assure la maîtrise d'ouvrage pour son propre compte. La filière de l'autopromotion s'oppose à la filière du "promoteur professionnel», où la maitrise d'ouvrage est assurée en vue d'une commercialisation et d'un profit financier. En matière de production immobilière résidentielle, la Belgique présente une particularité forte, celle de la prédominance de l'auto-promotion. Cette prédominance, sur laquelle nous reviendrons largement, détermine l'organisation des marchés fonciers résidentiels belges. En effet, lorsqu'il y a auto-promotion, les terrains supports des nouvelles constructions font souvent l'objet de deux échanges avant d'être construits. Préalablement à la vente aux ménages souhaitant bâtir, les terrains peuvent avoir fait l'objet d'une transaction entre le propriétaire initial et un promoteur foncier souhaitant lotir. Cette première transaction s'inscrit dans un marché des "gisements fonciers ", formule exprimant l'idée que la ressource naturelle «sol » correspond à la matière première d'un processus de production (J. Comby, 2003, p. 20).

Dans le cadre de cet article, nous viserons à analyser comment les secteurs de la promotion foncière et immobilière déterminent les modes d'utilisation du sol et la production des périphéries. Pour répondre à cet objectif, nous procéderons en trois parties. En première partie, nous présenterons brièvement le contexte spatial des nouvelles urbanisations résidentielles produites en Belgique. Nous y aborderons la thématique de l'offre foncière urbanisable, la réforme du Ruimtelijk Structuurplan Vlaanderen, la très forte aspiration des familles pour l'habitat individuel et certaines des raisons qui font de la Belgique un pays à la périurbanisation quasi généralisée. En deuxième partie, consacrée aux choix d'investissement des promoteurs fonciers, nous viserons à préciser pourquoi la périurbanisation belge s'accompagne d'une consommation d'espace extrêmement importante (J.-B. Jehin, 1998). Enfin, en troisième partie, la prise en compte de la promotion immobilière nous permettra d'expliquer la particularité belge de la forte prédominance de l'auto-promotion dans la constitution des stocks de logement. 


\section{Le contexte belge des nouveaux espaces résidentiels}

\section{A. Une tradition faiblement planificatrice et d'abondance en offre juridiquement urbanisable}

4 Rendre compte du caractère très soutenu de la périurbanisation belge nécessite de faire référence à la tradition faiblement planificatrice du pays. En matière de gestion foncière, cette tradition de faible planification se traduit notamment par une abondance en offre juridiquement urbanisable, c'est-à-dire l'offre telle que définie par le zonage fonctionnel et l'urbanisme réglementaire. Pour la Belgique, c'est très largement le dispositif des plans de secteur qui définit l'offre juridiquement urbanisable. Il s'agit de documents qui couvrent l'ensemble du pays, en différenciant les terrains ædificandi des terrains non ædificandi. En matière d'urbanisation résidentielle, ce sont les terrains inscrits au sein des zones d'habitat qui peuvent accueillir les nouveaux projets résidentiels. Tant en Flandre qu'en Wallonie, il est reconnu que les planificateurs ayant travaillé sur l'élaboration des plans de secteur ont très « généreusement » délimité les zones d'habitat. Les objectivations récentes menées sur les potentiels en terrains vierges toujours situés en leur sein attestent de cette générosité, de même d'ailleurs que l'absence de révision importante de ces zones depuis leur adoption dans le courant des décennies soixante-dix et quatre-vingt ${ }^{2}$.

5 La variable du ratio de disponibilité foncière, cartographiée en figure 1, permet de préciser ce constat selon lequel les zones d'habitat sont surdimensionnées par comparaison aux besoins en nouveaux logements. Elaborée dans le cadre d'une recherche sur la révision des plans de secteur wallons (O. Dubois et al., 2002, p. 28), cette variable se calcule via la confrontation entre, d'une part, les superficies morphologiquement urbanisées selon le cadastre et, d'autre part, les superficies inscrites en zone d'habitat aux plans de secteur. Le ratio de disponibilité foncière ne permet pas de déterminer les superficies exactes non encore urbanisées au sein des zones d'habitat. Toutefois, il permet d'apprécier le caractère plus ou moins saturé de ces zones. Globalement, plus l'indicateur est élevé, plus les terres à la fois vierges et juridiquement urbanisables sont limitées ${ }^{3}$. La cartographie de l'indicateur du ratio de disponibilité foncière met en évidence que la disponibilité en zones d'habitat dépend peu de la hiérarchie urbaine et de la distance aux noyaux urbains. Plutôt que d'observer des différences entre les "villes", les "périphéries" et les "campagnes", nous constatons des différences entre les provinces et entre les plans de secteur, ce qui semble s'expliquer par le jeu des rapports de force locaux lors de l'élaboration de ces documents réglementaires. 
Figure 1 : Ratio de disponibilité foncière en 2000.

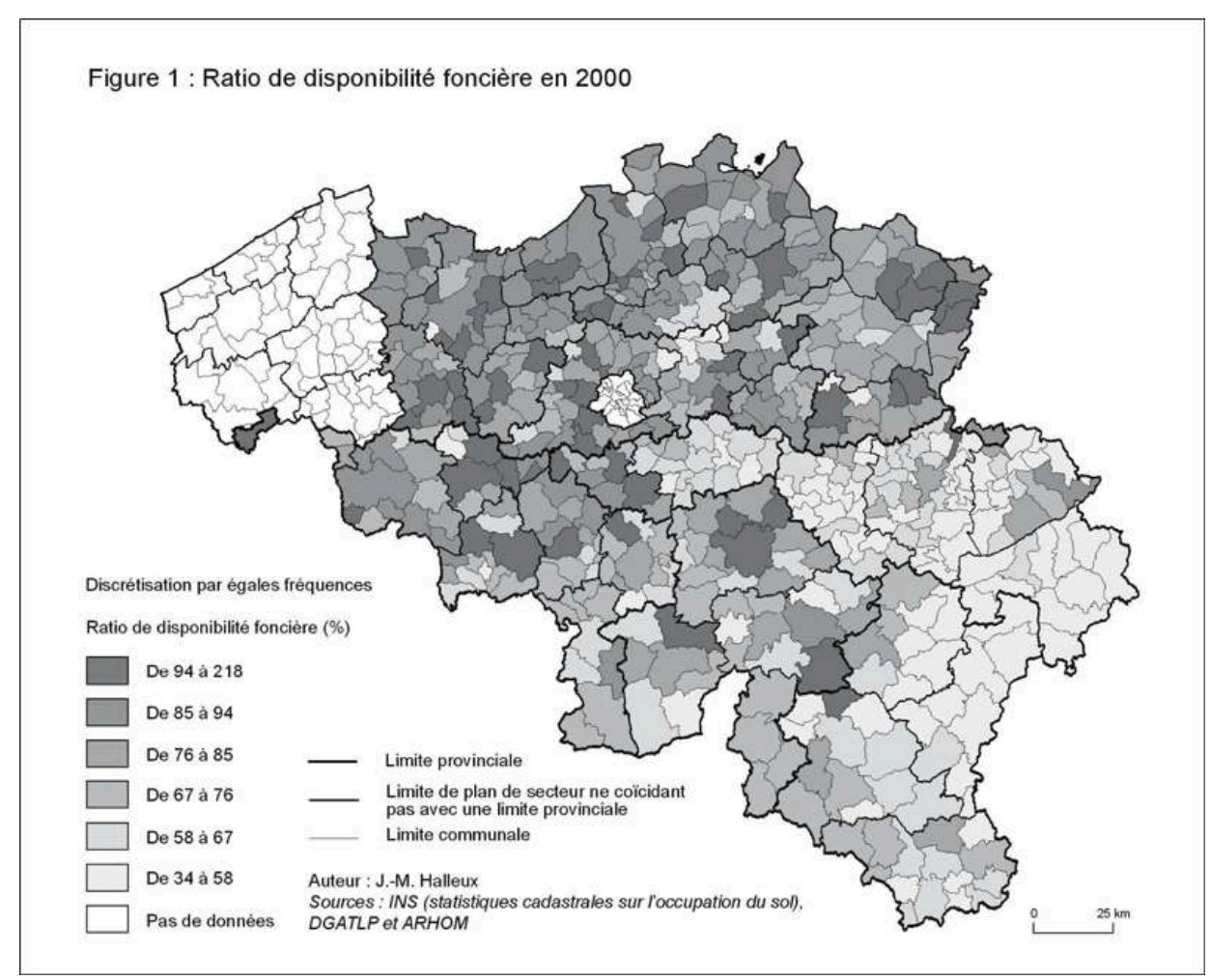

6 En matière d'offre foncière urbanisable, il est essentiel de discerner l'offre potentielle et l'offre effective (J.Comby et V. Renard, 1996). Pour un territoire de marché considéré, l'offre foncière potentielle correspond à la totalité des biens existants. Concernant l'offre juridique destinée à la production de nouveaux logements, il s'agit globalement de l'ensemble des terrains situés à l'intérieur des périmètres définis par les zones d'habitat. Quant à l'offre effective, elle correspond, à un moment donné, aux biens réellement disponibles sur le marché. En raison de la rétention fréquemment exercée par les propriétaires, l'offre effective ne correspond qu'à une part très réduite de l'offre potentielle. Lorsque les acteurs dont le rôle est de veiller à la sauvegarde de l'environnement naturel et construit (par exemple les administrations en charge de l'aménagement du territoire) évaluent les disponibilités foncières, ils font en général référence à l'ensemble des potentialités de l'offre juridiquement urbanisable. Par contre, lorsque des promoteurs fonciers ou immobiliers ont à lever l'obstacle foncier afin de mettre en œuvre des projets d'urbanisation, ils sont limités à la seule offre effective. Or, nombreux sont les contextes fonciers belges où l'on rencontre simultanément une surabondance d'offre juridique potentielle et une pénurie d'offre effective (J.-M. Halleux, 2002b).

7 En Belgique, l'intervention des pouvoirs publics sur les marchés fonciers est marginale et la pratique du lotissement public très peu courante. En raison du faible développement de la promotion foncière publique et de l'absence de dispositifs permettant une adéquation entre offre potentielle et offre effective, on observe une consommation des ressources foncières souvent en contradiction avec les objectifs fondamentaux de l'aménagement. En effet, la rétention pratiquée par de nombreux propriétaires, notamment pour certains terrains situés au sein même ou à proximité immédiate des agglomérations, engendre immanquablement un problème quant au 
positionnement des opérations d'urbanisation. Les terrains se libérant souvent de manière aléatoire, en fonction de la seule volonté des propriétaires et des lotisseurs privés, les sites les plus structurants pour l'urbanisation ne sont pas systématiquement disponibles sur le marché, ce qui éloigne les maitres d'ouvrages des noyaux urbains. Au final, il en résulte une périurbanisation très éclatée.

\section{B. La réforme du Ruimtelijk Structuurplan Vlaanderen}

Pour la Flandre, des modifications importantes furent apportées aux dispositifs de l'aménagement du territoire en 1997. C'est en effet à la fin de l'année 1997 que le document d'orientation dénommé Ruimtelijk Structuurplan Vlaanderen (Schéma de Structure Spatiale de la Flandre ou RSV) fut publié. Le RSV représente un changement notable par rapport à la politique de laisser-faire qui a prévalu jusque là. Il est vrai que face à une urbanisation de plus en plus éclatée et face à un territoire en forte perte de qualité, l'autorité flamande se devait de réagir.

9 A propos des enjeux de la nouvelle urbanisation résidentielle, le Schéma de Structure Spatiale de la Flandre vise notamment à limiter les nouvelles superficies dédiées à l'urbanisation résidentielle. C'est dans ce cadre que des seuils de densité minimale ont été prévus pour les nouveaux quartiers. Ces seuils sont de 25 habitations par hectare dans les zones urbaines et de 15 habitations par hectare en dehors des zones urbaines. En tenant compte de la superficie réservée aux voiries et aux espaces publics, ces normes juridiquement non contraignantes signifient que les superficies maximales des nouvelles parcelles devraient correspondre à 4 ares en zone urbaine et à 6,5 ares en zone non urbaine.

10 Contrairement aux attentes formulées par le secteur de la construction, le RSV ne prévoit pas d'élargir les zones juridiquement urbanisables. Bien que certains transferts de droits à bâtir puissent se produire entre sous-régions, l'administration flamande estime que les zones d'habitat actuelles sont suffisantes pour accueillir les développements démographiques. Pour les gestionnaires flamands en charge de l'aménagement du territoire, il n'est donc pas opportun d'élargir ces zones puisqu'une telle décision permettrait à la périurbanisation diffuse de se renforcer.

11 Pour opérationnaliser les objectifs du RSV, il est prévu que les plans de secteur flamands soient progressivement remplacés par de nouveaux dispositifs réglementaires établis aux niveaux provinciaux et communaux. Bien que le RSV ait été adopté voici maintenant huit ans, sa concrétisation tarde à se réaliser et les plans d'application chargés de le mettre en œuvre via les stades intermédiaires que constituent les schémas de structure provinciaux et communaux ne sont toujours pas opérationnels. Sans doute la difficulté de transformer les principes stratégiques de la parcimonie foncière en mesures réglementaires contraignantes explique-t-elle cette situation?

\section{Une très forte aspiration des familles pour l'habitat individuel et une périurbanisation presque généralisée}

Complémentairement à la tradition faiblement planificatrice, la forte préférence de la population belge pour la maison unifamiliale correspond également à un facteur explicatif de l'intense urbanisation périphérique qui caractérise le pays. Des enquêtes menées en matière de choix résidentiels pour la périphérie, il ressort que 
l'insatisfaction par rapport aux caractéristiques des logements correspond à la cause première de l'exurbanisation et de la périurbanisation (L. Brück et al., 2000). Le désir de quitter un intérieur trop petit, d'échanger un appartement pour une maison unifamiliale et l'absence de jardin sont des facteurs très souvent évoqués dans ce type d'enquête. Ces facteurs sont bien sûr étroitement corrélés à la logique des cycles de vie puisque le choix pour la périphérie est très fréquemment le fait du jeune couple avec enfants à la recherche d'un cadre résidentiel mieux adapté à la vie familiale d'un ménage agrandi. En Belgique, où l'acquisition immobilière est valorisée depuis plus d'un siècle, les motifs familiaux de la périurbanisation sont parfaitement corrélés avec cet autre facteur-clé qu'est le désir de devenir propriétaire. A ce propos, la sociologie nous apprend que, pour le jeune couple, l'accès à la propriété permet d'acquérir « une maison au sens de maisonnée, c'est-à-dire de créer un groupe social uni par les liens de l'alliance et de la parenté, que redoublent les liens de la cohabitation» (P. Bourdieu et al., 1990, p. 7).

13 Afin de recadrer les choix de la population belge pour l'habitat unifamilial, nous pouvons nous appuyer sur les comparaisons européennes présentées dans le tableau 1, où l'on observe que le parc de logements est en Belgique très majoritairement constitué de maisons unifamiliales. Renforcée par l'histoire urbaine et socio-politique du pays (C. Kesteloot et S. De Maesschalck, 2001; O. Dubois, 2002), l'aspiration profonde des populations belges pour l'habitat individuel semble s'inscrire dans un contexte culturel plus vaste, à savoir le modèle individualiste de l'Europe atlantique (J. Lévy, 1997, p. 142). Sur ce plan, la Belgique - à l'instar du Royaume-Uni, des Pays-Bas et du Danemark - se différencie des cultures rhénane et méditerranéenne, caractérisées par une plus forte disposition pour l'urbanité, par une meilleure acceptation de la vie en immeubles collectifs et, au final, par des parcs de logements où la maison unifamiliale est moins prédominante (J.-M. Halleux et al., 2002).

Tableau 1 : Comparaison internationale des parcs de logement.

\begin{tabular}{|l|l|l|l|l|}
\hline & $\begin{array}{l}\text { Part de la maison } \\
\text { unifamiliale }\end{array}$ & $\begin{array}{l}\text { Part de propriétaires } \\
\text { occupants }\end{array}$ & $\begin{array}{l}\text { Locataire du } \\
\text { secteur privé }\end{array}$ & $\begin{array}{l}\text { Locataire du } \\
\text { secteur public }\end{array}$ \\
\hline $\begin{array}{l}\text { Grande- } \\
\text { Bretagne }\end{array}$ & $79 \%$ & $67 \%$ & $10 \%$ & $23 \%$ \\
\hline Belgique & $73 \%$ & $65 \%$ & $28 \%$ & $6 \%$ \\
\hline Pays-Bas & $71 \%$ & $49 \%$ & $13 \%$ & $38 \%$ \\
\hline Danemark & $61 \%$ & $53 \%$ & $19 \%$ & $26 \%$ \\
\hline Portugal & $61 \%$ & $65 \%$ & $15 \%$ & $3 \%$ \\
\hline Norvège & $58 \%$ & $59 \%$ & $19 \%$ & $3 \%$ \\
\hline France & $56 \%$ & $54 \%$ & $22 \%$ & $17 \%$ \\
\hline Suède & $54 \%$ & $42 \%$ & $29 \%$ & $23 \%$ \\
\hline Autriche & $48 \%$ & $50 \%$ & $10 \%$ \\
\hline
\end{tabular}




\begin{tabular}{|l|l|l|l|l|}
\hline Allemagne & $46 \%$ & $39 \%$ & $37 \%$ & $24 \%$ \\
\hline Espagne & $36 \%$ & $85 \%$ & $14 \%$ & $1 \%$ \\
\hline Italie & $32 \%$ & $70 \%$ & $20 \%$ & $4 \%$ \\
\hline Suisse & $21 \%$ & $31 \%$ & $67 \%$ & $2 \%$ \\
\hline
\end{tabular}

Source : P. de la Morvonnais, 1998

Référence : début de la décennie 1990.

Si l'intense périurbanisation résidentielle que l'on observe en Belgique résulte sans conteste de l'aspiration des populations pour l'habitat individuel, le choix pour la périphérie qu'expriment de très nombreux ménages est également le résultat des contraintes induites par le fonctionnement des marchés du logement. Parmi ces contraintes, nous trouvons notamment la difficulté d'accéder à une offre immobilière qui soit à la fois positionnée au sein des espaces urbains centraux, de qualité, et financièrement accessible. D'autres contraintes résident dans les très hauts niveaux de prix pratiqués au sein des premières couronnes périurbaines et dans l'obligation pour de nombreux ménages de s'éloigner en lointaine périphérie afin d'accéder à une offre foncière financièrement accessible (O. Dubois et J.-M. Halleux, 2003). La faible disponibilité en offre foncière effective semble également déterminer les choix résidentiels pour la périurbanisation étalée. En effet, la configuration des zones d'habitat n'ayant pas été sensiblement modifiée depuis l'adoption des plans de secteur, il y a maintenant plus de deux décennies pour la plupart d'entre eux, la disponibilité en offre effective se réduit au fur et à mesure des nouvelles occupations urbaines. Au sein des régions ayant été les moins bien dotées en zones d'habitat, de réelles pénuries en offre effective sont désormais observées, ce qui, au final, oblige les ménages à considérablement élargir leur zone de prospection foncière afin d'accéder à l'acquisition d'une parcelle constructible (J.-M. Halleux, 2005).

Conjuguée à la densité du réseau urbain et à une tradition de navettes antérieure à la banalisation de la conduite automobile, l'intense périurbanisation diffuse qui caractérise aujourd'hui la Belgique pousse les observateurs à assimiler l'ensemble du territoire national à une vaste conurbation qu'articulent dix-sept complexes résidentiels urbains (H. Van der Haegen et al., 1998), un réseau très dense de petites villes et différents pôles urbains étrangers. Concernant les territoires situés entre la Lorraine belge et le sillon wallon, où l'urbanisation est historiquement moins intense, l'on découvre aujourd'hui que le peuplement relativement soutenu qui caractérise cette partie du pays ne peut pas non plus être dissocié de l'influence urbaine. En effet, les flux migratoires qui alimentent ce peuplement ne se démarquent pas du modèle périurbain et ce sont tout d'abord les choix résidentiels de familles avec enfants provenant des grandes agglomérations wallonnes ou des petites villes locales qui expliquent ce phénomène (T. Eggerick et $C$. Capron, 2001). 


\section{Le rôle de la promotion foncière en matière de consommation d'espace}

\section{A. Les critères auxquels les promoteurs fonciers font référence lorsqu'ils définissent la superficie des lots à bâtir}

Connaissant les principales caractéristiques du contexte spatial analysé, il nous est maintenant possible d'analyser le comportement et les choix d'investissement des promoteurs qui y sont actifs. Nous débuterons cette analyse par la prise en compte de la promotion foncière, afin d'appréhender l'évolution actuelle vers le desserrement, c'est-à-dire la production de lots constructibles dont les superficies sont en général bien vastes en comparaison des parcelles bâties préexistantes (V. Fouchier, 2001). Nous avons pour ce faire choisi de nous intéresser à la superficie moyenne des terrains qui sont échangés sur le marché des parcelles constructibles et aux critères auxquels les promoteurs fonciers font référence lorsqu'ils définissent la superficie des lots qu'ils souhaitent commercialiser. Pour la Belgique, il n'existe malheureusement pas de sources statistiques distinguant explicitement le marché des lots et le marché des gisements. Néanmoins, la prise en compte de la surface des parcelles échangées permet d'approcher cette différenciation et, dans ce cadre, les données disponibles nous ont permis de calculer un indicateur de superficie moyenne pour les seules transactions correspondant à des parcelles dont la surface est inférieure à $1500 \mathrm{~m}^{2}$.

17 Le tableau 2 illustre une caractéristique importante du prix des terres à bâtir : leur «marginalité décroissante» en fonction de la superficie (P.F. Colwell et H.J. Munneke, 1997). Globalement, lorsque la superficie des terrains échangés s'accroît, l'on observe simultanément une croissance du prix par transaction et une décroissance du prix moyen par mètre carré. Deux explications sont nécessaires pour rendre compte de cette caractéristique : d'une part, la différenciation entre le marché des gisements et le marché des lots constructibles; d'autre part, l'influence de la superficie des lots sur les utilités retirées par les demandeurs et sur les coûts de production assumés par les promoteurs. Il ressort de la marginalité décroissante en fonction de la superficie que le promoteur foncier minimisera la superficie des lots qu'il produit s'il tient à maximiser son chiffre d'affaires et son profit. Pour autant, cette minimisation doit tenir compte d'autres facteurs que la seule maximisation du chiffre d'affaires et, sur ce plan, il est nécessaire de faire référence au risque d'une commercialisation difficile. En fait, le lotisseur expérimenté visera à maximiser son chiffre d'affaires en tenant compte de la contrainte de la commercialisation ou, en d'autres termes, il visera à produire les lots commercialisables les plus resserrés possible.

Tableau 2 : Influence des classes de superficie sur les caractéristiques des transactions foncières (Flandre et Wallonie pour l'année 2000).

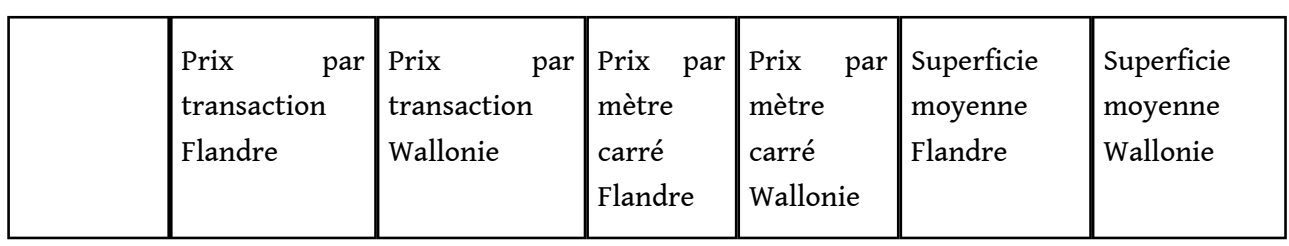




\begin{tabular}{|l|l|l|l|l|l|l|}
\hline $\begin{array}{l}\text { De } \quad 180 \quad \text { à } \\
360 \mathrm{~m}^{2}\end{array}$ & $27282 €$ & $7908 €$ & $97 €$ & $29 €$ & $282 \mathrm{~m}^{2}$ & $271 \mathrm{~m}^{2}$ \\
\hline $\begin{array}{l}\text { De } 360 \quad \text { à } \\
720 \mathrm{~m}^{2}\end{array}$ & $42844 €$ & $16764 €$ & $77 €$ & $30 €$ & $555 \mathrm{~m}^{2}$ & $555 \mathrm{~m}^{2}$ \\
\hline $\begin{array}{l}\text { De } 720 \text { à } \\
1500 \mathrm{~m}^{2}\end{array}$ & $66750 €$ & $25345 €$ & $66 €$ & $23 €$ & $1006 \mathrm{~m}^{2}$ & $1085 \mathrm{~m}^{2}$ \\
\hline $\begin{array}{l}\text { Plus de } \\
1500 \mathrm{~m}^{2}\end{array}$ & $133534 €$ & $51966 €$ & $37 €$ & $15 €$ & $3580 \mathrm{~m}^{2}$ & $3372 \mathrm{~m}^{2}$ \\
\hline Moyenne & $\mathbf{6 3 6 8 4 €}$ & $\mathbf{3 0 1 4 0 €}$ & $\mathbf{6 5} €$ & $\mathbf{2 1} €$ & $\mathbf{9 8 2} \mathbf{~ m}^{\mathbf{2}}$ & $\mathbf{1 4 4 4 \mathbf { ~ m } ^ { 2 }}$ \\
\hline
\end{tabular}

Source : STADIM

Les tests statistiques que nous avons réalisés afin d'expliquer la distribution spatiale de la superficie moyenne des terrains de moins de $1500 \mathrm{~m}^{2}$ confirment l'hypothèse selon laquelle les lotisseurs cherchent à produire les lots commercialisables les plus resserrés possible. Pour ces tests, menés via la technique de la régression multiple, l'échelle de traitement des données correspond à l'ensemble du pays. Que les données soient agrégées au niveau des communes ou au niveau des bassins d'emploi ${ }^{4}$, il ressort que les deux variables du prix et du ratio de disponibilité ont un impact statistiquement significatif sur les superficies moyennes (graphiques 1 et 2). Ces résultats peuvent directement s'interpréter en référence aux choix et aux contraintes qui pèsent sur la demande. A propos de l'influence des prix, le caractère significatif de la relation indique que des prix élevés forcent les ménages à limiter leur demande pour les grands terrains, ce qui, en conséquence, va permettre aux lotisseurs d'alimenter le marché avec des lots resserrés. Pour la relation entre la disponibilité en offre juridique potentielle et la superficie, nous pouvons déduire qu'un accès plus difficile au marché foncier est un élément qui pousse également les ménages à accepter des lots resserrés et, parallèlement, que le renforcement de la concurrence pour l'acquisition des gisements pousse les lotisseurs à limiter la superficie des parcelles offertes sur le marché des lots constructibles. Cette idée est corroborée par l'analyse cartographique relative à la superficie moyenne des lots constructibles. Une telle analyse souligne que les territoires caractérisés par les parcelles les plus vastes remplissent certaines conditions concernant la topographie et l'offre juridiquement urbanisable. Il s'agit de régions caractérisées par un relief de plateau relativement peu tourmenté et par de fortes disponibilités en zones d'habitat (notamment en Ardenne centrale, en Lorraine belge et dans le Condroz) ; là où les limitations de la topographie et de l'offre juridique sont faibles, le lotisseur qui prendrait le risque de réduire la superficie des parcelles en vue d'accroître son chiffre d'affaires serait incapable d'attirer la demande si ses concurrents choisissent d'alimenter le marché avec des lots plus vastes. 
Graphique 1 : Variabilité de la superficie des terrains à bâtir de moins de $1500 \mathrm{~m}^{2}$ en fonction du ratio de disponibilité foncière.

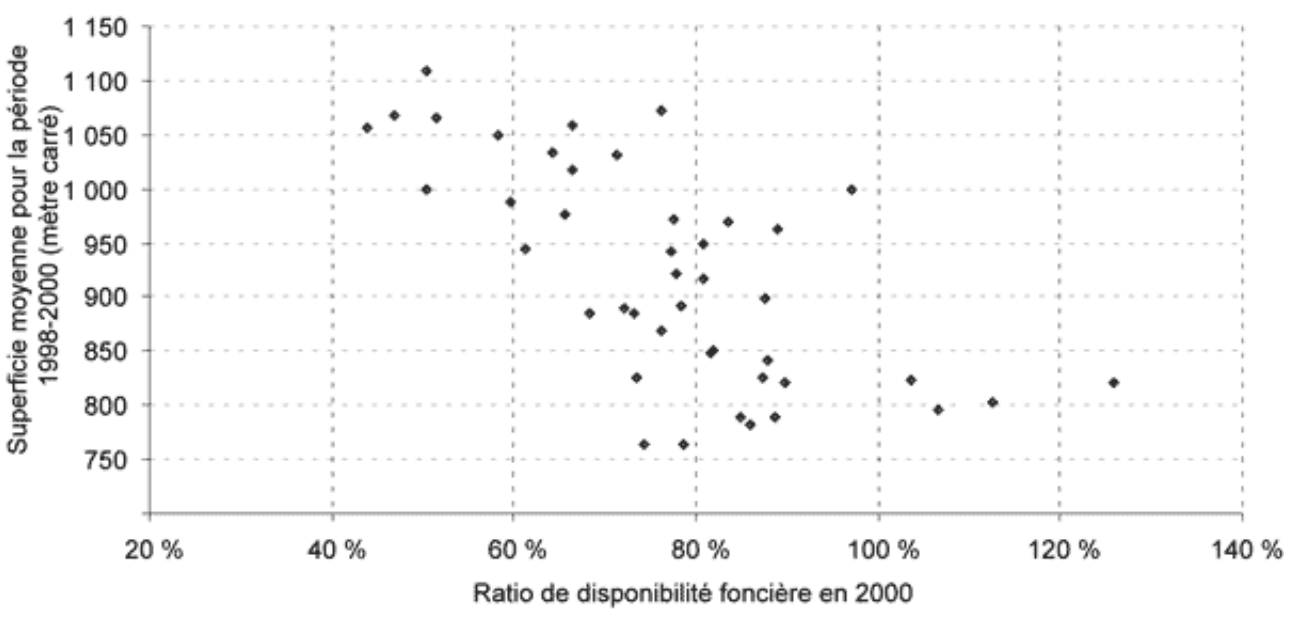

Ensemble de la Belgique à l'échelle des bassins d'emploi (sauf Flandre occidentale et BruxellesCapitale).

Sources: INS (statistiques cadastrales sur l'occupation du sol), DGATLP, ARHOM et STADIM.

Graphique 2: Variabilité de la superficie des terrains à bâtir de moins de $1500 \mathrm{~m}^{2}$ en fonction de leur prix.

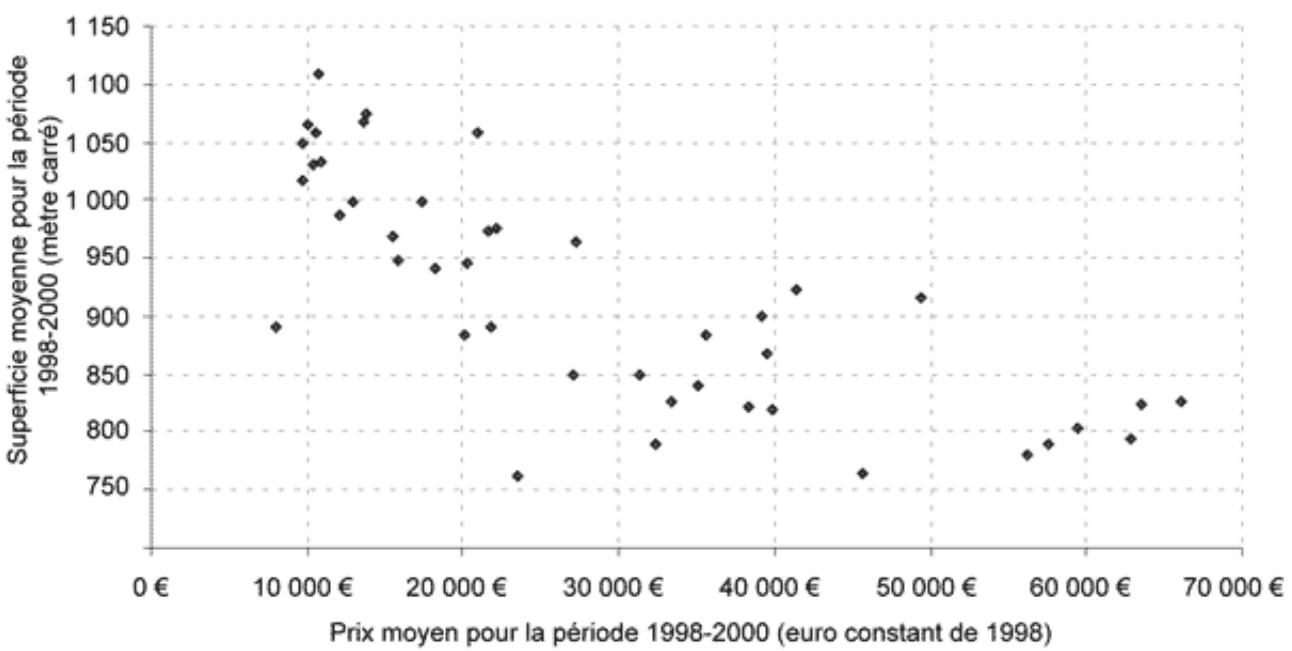

Ensemble de la Belgique à l'échelle des bassins d'emploi (sauf Flandre occidentale et BruxellesCapitale).

Source : STADIM.

\section{B. Observation des disparités spatiales et des tendances évolutives}

Pour la période 1998-1999-2000, la superficie moyenne des terrains à bâtir (terrains de plus de $1500 \mathrm{~m}^{2}$ exclus) est de $731 \mathrm{~m}^{2}$ pour l'ensemble de la Belgique. Pour les trois Régions de Flandre, de Wallonie et de Bruxelles-Capitale, les moyennes sont respectivement de $684 \mathrm{~m}^{2}, 831 \mathrm{~m}^{2}$ et $409 \mathrm{~m}^{2}$. La faible superficie observée pour Bruxelles tient aux caractéristiques de sa production immobilière. Alors que les marchés fonciers wallons et flamands demeurent dominés par des transactions dont la finalité correspond à l'édification de maisons unifamiliales, les transactions foncières bruxelloises sont par contre menées avec l'objectif de commercialiser des immeubles de 
bureaux ou de logements collectifs. Concernant la différence entre la Flandre et la Wallonie, il est intéressant d'observer son récent renforcement, comme l'illustre le graphique 3. En Wallonie, la tendance vers le desserrement s'accentue et les parcelles échangées à la fin de la décennie quatre-vingt-dix sont plus vastes encore que les parcelles échangées à la fin de la décennie quatre-vingt. Au nord de la frontière linguistique, il se produit à l'inverse une limitation du desserrement et l'indicateur de la superficie moyenne par parcelle tend à se réduire. Graphique 3 : Évolution de la superficie moyenne pour les terrains à bâtir de moins de $1500 \mathrm{~m}^{2}$ en
Flandre et en Wallonie.

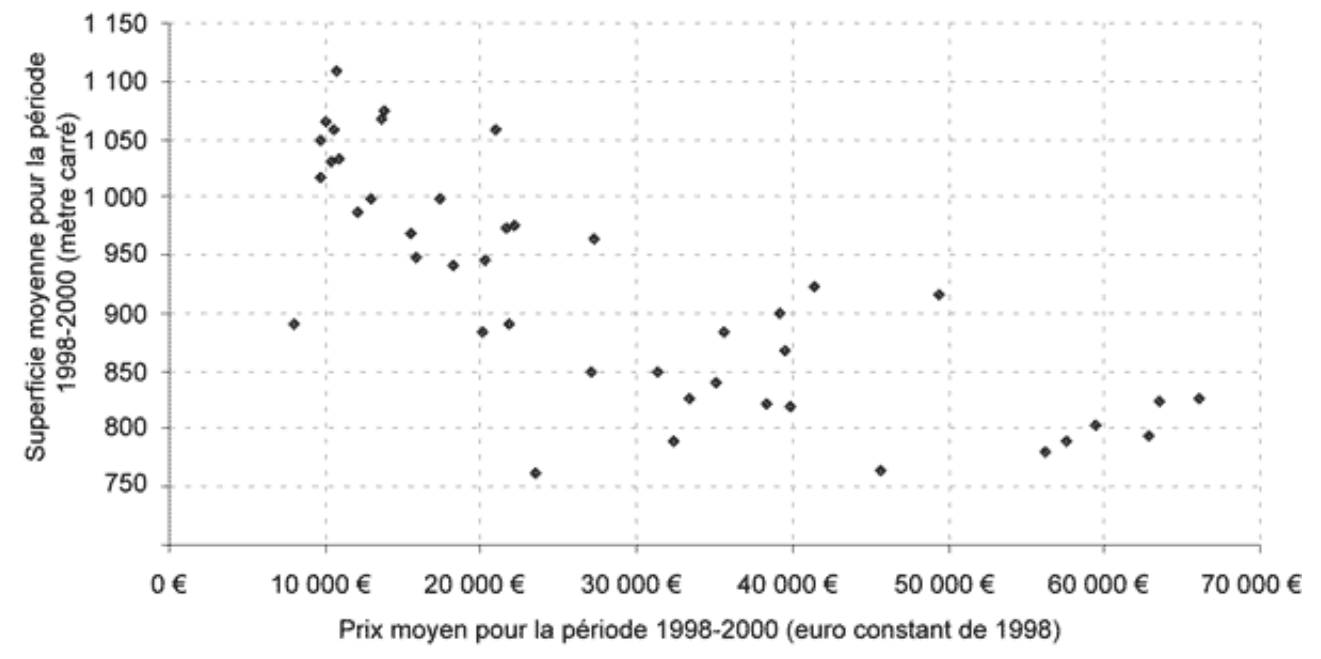

Source : STADIM

En Flandre, la réduction de la superficie moyenne par parcelle s'enclenche à partir de 1998, soit une année après la publication du Ruimtelijk Structuurplan Vlaanderen, ce qui confirme l'influence de la disponibilité en offre sur le caractère plus ou moins resserré des lots constructibles. Rappelons que le RSV prévoit de ne pas permettre l'élargissement des zones urbanisables et de progressivement appliquer une politique foncière plus stricte. Bien que ce projet territorial ne se soit pas accompagné de modifications concrètes en matière d'aménagement réglementaire et opérationnel, le contexte tendu des marchés fonciers flamands explique pourquoi il les a profondément influencés. En effet, pour les promoteurs fonciers, la publication de ce projet de développement territorial s'est sans doute traduite par une crainte renforcée d'aller vers la pénurie en offre foncière lotissable. Or, les stratégies de commercialisation des lotisseurs étant dépendantes de la disponibilité en offre et de leur plus ou moins grande facilité à se positionner sur le marché des gisements, il est logique que la crainte d'une pénurie les ait poussé à alimenter le marché avec des lots resserrés. Pour expliquer la réduction de la superficie des lots observée entre 1997 et 2000, il faut également invoquer la déclaration d'intention du RSV quant aux seuils de densité minimale pour les nouveaux quartiers (parcelles maximales de $400 \mathrm{~m}^{2}$ par habitation urbaine et de $650 \mathrm{~m}^{2}$ pour les autres). Bien que non contraignante, il est probable que la parution de cette norme ait renforcé la tendance vers la production de lots resserrés.

Les données rassemblées dans le tableau 3 permettent de préciser les grandes tendances relatives à l'emprise foncière des nouveaux terrains à bâtir. Ce tableau s'appuie sur un classement des communes, en fonction de la typologie établie sur base 
du phénomène des régions urbaines ${ }^{5}$ (H. Van der Haegen et al., 1998) et en fonction, d'autre part, de la différenciation Flandre - Wallonie. La première colonne du tableau 3 désigne les groupements de communes considérés. En deuxième colonne, il est rendu compte de la superficie moyenne des lots à bâtir échangés entre 1998 et 2000. En troisième colonne, nous relativisons l'indicateur absolu de la superficie moyenne par la moyenne nationale $\left(731 \mathrm{~m}^{2}\right)$. Les quatrième et cinquième colonnes renseignent sur la tendance évolutive observée entre 1988 et 2000 (nous reprenons ici l'évolution annuelle de la superficie, inférée sur base d'un ajustement linéaire prenant en compte les 13 informations correspondant aux 13 années écoulées entre 1988 et 2000), en valeur absolue et en valeur relative respectivement.

Tableau 3 : Superficie moyenne pour les lots à bâtir sans les terrains de plus de 1500 mètres carrés.

\begin{tabular}{|c|c|c|c|c|}
\hline & \multicolumn{2}{|c|}{$\begin{array}{l}\text { Superficie moyenne pour la période } \\
\text { 1998-1999-2000 }\end{array}$} & \multicolumn{2}{|c|}{$\begin{array}{l}\text { Évolution annuelle de } 1988 \text { à 2000. Calcul } \\
\text { par ajustement linéaire }\end{array}$} \\
\hline & $\begin{array}{l}\text { Valeur } \\
\text { absolue }\end{array}$ & $\begin{array}{l}\text { Pourcentage de la } \\
\text { moyenne nationale }\end{array}$ & Évolution absolue & $\begin{array}{l}\text { Évolution } \\
\text { relative }\end{array}$ \\
\hline Belgique & $731 \mathrm{~m}^{2}$ & $100 \%$ & $1,5 \mathrm{~m}^{2}$ & $0,2 \%$ \\
\hline Bruxelles-Capitale & $409 \mathrm{~m}^{2}$ & $56 \%$ & $-1,3 \mathrm{~m}^{2}$ & $-0,3 \%$ \\
\hline Flandre & $684 \mathrm{~m}^{2}$ & $94 \%$ & $-1,7 \mathrm{~m}^{2}$ & $-0,2 \%$ \\
\hline $\begin{array}{l}\text { Villes centrales en } \\
\text { Flandre }\end{array}$ & $578 \mathrm{~m}^{2}$ & $79 \%$ & $1,3 \mathrm{~m}^{2}$ & $0,2 \%$ \\
\hline $\begin{array}{l}\text { Agglomération flamande } \\
\text { de Bruxelles }\end{array}$ & $586 \mathrm{~m}^{2}$ & $80 \%$ & $-1,8 \mathrm{~m}^{2}$ & $-0,3 \%$ \\
\hline $\begin{array}{l}\text { Agglomération en } \\
\text { Flandre }\end{array}$ & $592 \mathrm{~m}^{2}$ & $81 \%$ & $-0,3 \mathrm{~m}^{2}$ & $-0,1 \%$ \\
\hline $\begin{array}{l}\text { Banlieue flamande de } \\
\text { Bruxelles }\end{array}$ & $693 \mathrm{~m}^{2}$ & $95 \%$ & $-6,4 \mathrm{~m}^{2}$ & $-0,8 \%$ \\
\hline Banlieue en Flandre & $717 \mathrm{~m}^{2}$ & $98 \%$ & $-3,6 \mathrm{~m}^{2}$ & $-0,5 \%$ \\
\hline $\begin{array}{l}\text { ZRMA flamande de } \\
\text { Bruxelles }\end{array}$ & $707 \mathrm{~m}^{2}$ & $97 \%$ & $-3,8 \mathrm{~m}^{2}$ & $-0,5 \%$ \\
\hline ZRMA en Flandre & $686 \mathrm{~m}^{2}$ & $94 \%$ & $-3,1 \mathrm{~m}^{2}$ & $-0,4 \%$ \\
\hline Hors CRU en Flandre & $731 \mathrm{~m}^{2}$ & $100 \%$ & $-2,1 \mathrm{~m}^{2}$ & $-0,3 \%$ \\
\hline Wallonie & $831 \mathrm{~m}^{2}$ & $114 \%$ & $5,2 \mathrm{~m}^{2}$ & $0,7 \%$ \\
\hline $\begin{array}{l}\text { Villes centrales en } \\
\text { Wallonie }\end{array}$ & $696 \mathrm{~m}^{2}$ & $95 \%$ & $1,3 \mathrm{~m}^{2}$ & $0,2 \%$ \\
\hline
\end{tabular}




\begin{tabular}{|c|c|c|c|c|}
\hline $\begin{array}{l}\text { Agglomération wallonne } \\
\text { de Bruxelles }\end{array}$ & $657 \mathrm{~m}^{2}$ & $90 \%$ & $-3,4 m^{2}$ & $-0,5 \%$ \\
\hline $\begin{array}{l}\text { Agglomération en } \\
\text { Wallonie }\end{array}$ & $712 \mathrm{~m}^{2}$ & $97 \%$ & $3,8 \mathrm{~m}^{2}$ & $0,6 \%$ \\
\hline $\begin{array}{l}\text { Banlieue wallonne de } \\
\text { Bruxelles }\end{array}$ & $823 \mathrm{~m}^{2}$ & $113 \%$ & $-3,0 \mathrm{~m}^{2}$ & $-0,3 \%$ \\
\hline Banlieue en Wallonie & $880 \mathrm{~m}^{2}$ & $120 \%$ & $3,5 \mathrm{~m}^{2}$ & $0,4 \%$ \\
\hline $\begin{array}{l}\text { ZRMA wallonne de } \\
\text { Bruxelles }\end{array}$ & $881 \mathrm{~m}^{2}$ & $121 \%$ & $-1,1 \mathrm{~m}^{2}$ & $-0,1 \%$ \\
\hline ZRMA en Wallonie & $888 \mathrm{~m}^{2}$ & $121 \%$ & $3,6 \mathrm{~m}^{2}$ & $0,4 \%$ \\
\hline Hors CRU en Wallonie & $873 \mathrm{~m}^{2}$ & $119 \%$ & $7,9 \mathrm{~m}^{2}$ & $1,0 \%$ \\
\hline $\begin{array}{l}\text { Complexes résidentiels } \\
\text { urbains }\end{array}$ & & & & \\
\hline Bruxelles & $698 \mathrm{~m}^{2}$ & $96 \%$ & $-1,1 \mathrm{~m}^{2}$ & $-0,2 \%$ \\
\hline $\begin{array}{l}\text { Partie flamande pour } \\
\text { Bruxelles }\end{array}$ & $665 \mathrm{~m}^{2}$ & $91 \%$ & $-3,4 \mathrm{~m}^{2}$ & $-0,5 \%$ \\
\hline $\begin{array}{l}\text { Partie wallonne pour } \\
\text { Bruxelles }\end{array}$ & $831 \mathrm{~m}^{2}$ & $114 \%$ & $-0,1 \mathrm{~m}^{2}$ & $0,0 \%$ \\
\hline Anvers & $659 \mathrm{~m}^{2}$ & $90 \%$ & $-1,7 \mathrm{~m}^{2}$ & $-0,3 \%$ \\
\hline Liège & $796 \mathrm{~m}^{2}$ & $109 \%$ & $7,9 \mathrm{~m}^{2}$ & $1,1 \%$ \\
\hline Gand & $700 \mathrm{~m}^{2}$ & $96 \%$ & $-2,1 \mathrm{~m}^{2}$ & $-0,3 \%$ \\
\hline Charleroi & $815 \mathrm{~m}^{2}$ & $111 \%$ & $7,1 \mathrm{~m}^{2}$ & $1,0 \%$ \\
\hline
\end{tabular}

Source : STADIM.

Pour l'ensemble de la Belgique, la tendance globale est à l'accentuation du desserrement. Entre 1988 et 2000, la croissance tendancielle est de 1,5 mètres carrés par année (croissance relative de 0,2 \%). Bien sûr, l'évolution est fortement différenciée en fonction des appartenances régionales et les calculs confirment l'opposition entre l'accentuation du desserrement wallon (croissance annuelle de $+5,2 \mathrm{~m}^{2}$ ) et la limitation $\mathrm{du}$ desserrement flamand (décroissance annuelle de $-1,7 \mathrm{~m}^{2}$ ). Pour la Flandre, la tendance à la réduction de la superficie des parcelles concerne les agglomérations, les banlieues périurbaines, les zones résidentielles des migrants alternants et les territoires situés en dehors des complexes résidentiels urbains. Seules les villes centrales, c'est-à-dire le groupement de communes où les parcelles sont les moins vastes $\left(578 \mathrm{~m}^{2}\right.$, soit $79 \%$ de la moyenne nationale), font exception à la règle. La limitation du desserrement flamand étant inexistante dans les villes centrales et très 
modérée dans les agglomérations, il semble exister un effet butoir, qui se manifeste aux alentours des $600 \mathrm{~m}^{2}$.

En Wallonie, l'accentuation du desserrement concerne les différents types de communes, mais suivant des modalités différentes. L'évolution est particulièrement affirmée pour le groupement des communes n'appartenant pas aux complexes résidentiels urbains $\left(+7,9 \mathrm{~m}^{2}\right.$ par an). Cela semble attester de la diffusion du modèle de la très vaste parcelle en dehors des territoires périurbains traditionnels. La prise en compte spécifique des communes wallonnes du complexe résidentiel urbain bruxellois témoigne d'une tendance localisée à la réduction de la superficie des parcelles, à l'image de la situation flamande. Pour expliquer ce constat, il peut être fait référence aux prix élevés qui caractérisent ces territoires sous orbite bruxelloise et à la progressive saturation des zones d'habitat. Après plusieurs décades d'une périurbanisation très gourmande en ressource foncière, l'offre effective s'y raréfie et les promoteurs fonciers peuvent, sans risque pour leur commercialisation, alimenter le marché avec des parcelles de plus en plus réduites. Pour le complexe résidentiel urbain (CRU) de Bruxelles, la limitation du desserrement concerne l'agglomération $\left(-3,4 \mathrm{~m}^{2}\right.$ par an), la banlieue $\left(-3,0 \mathrm{~m}^{2}\right.$ par an) et, dans une moindre mesure, la zone résidentielle des migrants alternants (ZRMA) $\left(-1,1 \mathrm{~m}^{2}\right.$ par an). Toujours à propos de la partie wallonne du CRU bruxellois, il est frappant que la limitation du desserrement soit quasi inexistante lorsque les différentes couronnes sont simultanément prises en compte ($0,1 \mathrm{~m}^{2}$ par an). C'est ici au phénomène d'étalement de la périurbanisation qu'il faut faire référence. En effet, le différentiel entre l'évolution globale et les évolutions des différentes couronnes résulte d'une réduction des échanges au sein de l'agglomération et de la banlieue et, à l'inverse, d'une activation du marché au sein des territoires plus périphériques de la ZRMA.

\section{La promotion immobilière en maisons unifamiliales et la prédominance de l'auto-promotion}

\section{A. La prédominance de l'auto-promotion : une spécificité belge}

Par rapport aux pays voisins intégrés dans le contexte Atlantique-Nord de la maison unifamiliale, la Belgique présente une spécificité forte, correspondant à l'implication des particuliers dans le processus de développement. A l'exception notable de la Belgique, l'auto-promotion est très peu présente dans les pays où le parc de logements est majoritairement constitué de maisons unifamiliales. En effet, le Royaume-Uni, les Pays-Bas et le Danemark se caractérisent par une forte représentation de la filière du promoteur professionnel. En Belgique, à l'inverse, la filière de l'auto-promotion est très développée. Des principaux pays occidentaux pris en compte par le graphique 4, c'est en fait à ce pays que revient la palme de l'auto-promotion ${ }^{6 !}$ Plutôt que d'acquérir l'habitation déjà construite par un promoteur professionnel, comme ses homologues britanniques, néerlandais ou danois, le ménage belge désireux de bénéficier d'une construction unifamiliale neuve est donc généralement maitre d'ouvrage: il «fait construire ». Pour les maîtres d'œuvre, les candidats-bâtisseurs ${ }^{7}$ ont le choix entre deux grandes possibilités. Certains font appel à une entreprise générale de construction et bénéficient ainsi d'un produit communément dénommé "clé sur porte ». D'autres 
préfèrent solliciter l'intervention de différentes entreprises spécialisées en s'appuyant sur la coordination d'un architecte.

Graphique 4 : Part de l'auto-promotion dans la production de nouveaux logements (décennie 1980).

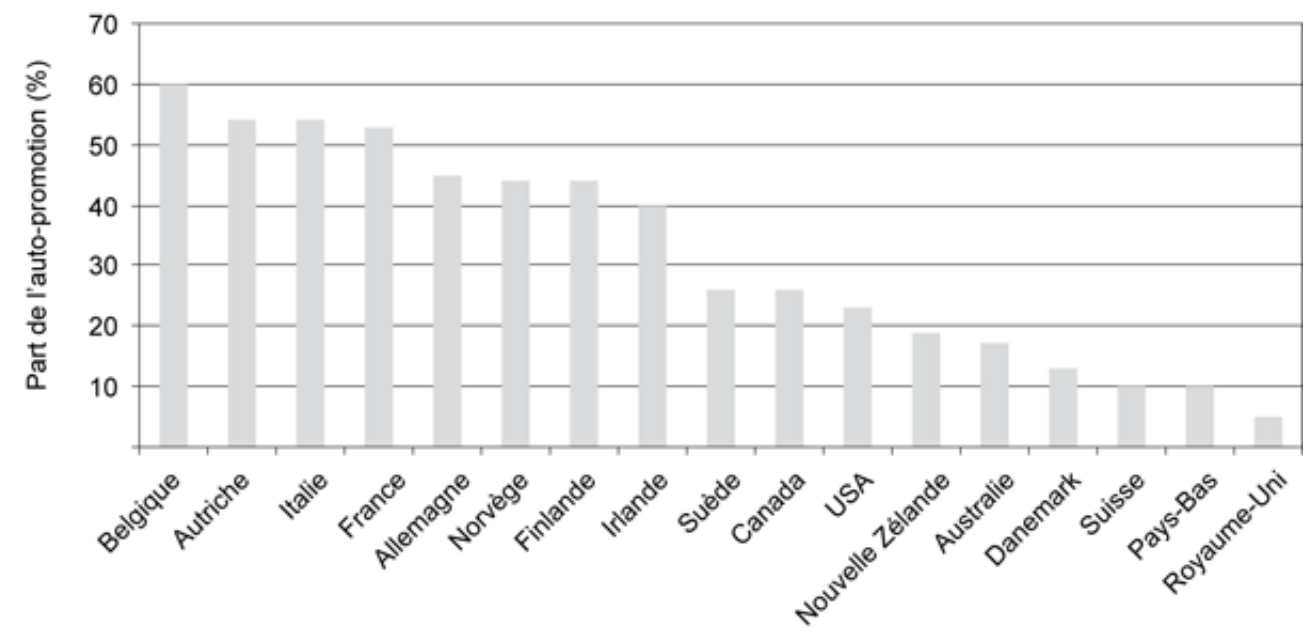

Source : S.S. Duncan et A. Rowe, 1993, p. 1331

Expliquer la spécificité belge de l'auto-promotion et rendre compte des disparités nationales quant à la ventilation entre la filière du promoteur professionnel et la filière de l'auto-promotion nécessite de faire référence aux relations systémiques entre les mécanismes de la production foncière et le caractère plus ou moins rigoureux de la planification spatiale. En effet, il semble que la maîtrise d'ouvrage des particuliers futurs occupants se répande là où la planification est peu rigoureuse et que, à l'inverse, la rigueur foncière pousse les constructeurs professionnels à intervenir en tant que maître d'ouvrage.

À notre connaissance, il n'existe pas de travaux spécifiquement consacrés aux relations entre la planification foncière et la faiblesse de l'auto-promotion au Danemark et aux Pays-Bas. Le cas sans doute relativement proche du Royaume-Uni est par contre bien documenté. Il en ressort que le poids de la filière "promoteur " y résulte de la faible disponibilité en offre potentielle juridiquement urbanisable (M. Ball, 2003). Plus précisément, l'élément crucial correspondrait à l'incapacité des particuliers à accéder au marché foncier (S.S. Duncan et A. Rowe, 1993, p. 1342). A l'image des configurations néerlandaises et danoises, les mécanismes de la production foncière s'accompagnent au Royaume-Uni d'une limitation stricte de l'offre juridique potentielle et d'une concentration de l'offre effective. Selon Duncan et Rowe, cette concentration permet aux constructeurs de bénéficier d'économies d'échelle, ce qui les pousse à intervenir directement sur les marchés fonciers afin d'assurer conjointement la viabilisation foncière et la construction immobilière. Un premier type d'économie d'échelle est lié à l'acquisition du terrain-support, moins coûteuse à gérer par unité de logement si l'offre est concentrée. La grille explicative des économies d'échelle s'applique également à la gestion des chantiers. En effet, la décroissance du coût marginal par logement avec l'augmentation du nombre de constructions incite les promoteurs-constructeurs à profiter de la possibilité des chantiers groupés offerte par la concentration des disponibilités foncières. 
Rendre compte des complexes relations systémiques entre les contextes nationaux et les modalités de la production immobilière résidentielle nécessite de considérer d'autres explications que les seuls mécanismes de la production foncière. Pour le Royaume-Uni, on voit par exemple que certains dispositifs de l'urbanisme réglementaire limitent explicitement les possibilités de l'auto-promotion. Dans ce pays, l'on observe aussi que le poids de la filière promoteur et la prégnance des traditions influencent l'attitude des institutions financières qui, bien souvent, rechignent à accorder un crédit hypothécaire aux particuliers qui souhaiteraient se lancer dans la maitrise d'ouvrage pour leur propre compte (S.S. Duncan et A. Rowe, 1993, p. 1343). La faiblesse de la filière de l'auto-promotion observée outre-Manche est également à mettre en relation avec la structure du secteur de la construction britannique, dont le segment de la nouvelle habitation unifamiliale est aujourd'hui dominé par un nombre limité de promoteurs-constructeurs importants (G. Bramley et al., 1995, p. 88).

Nous l'avons commenté ci-dessus, la politique "généreuse» en matière d'offre juridique potentielle se traduit en Belgique par une faible concentration de l'offre effective. Dans ce contexte de dispersion, le secteur de la construction résidentielle ne semble guère attiré par une intervention directe sur les gisements fonciers. Parallèlement, les particuliers sont souvent aptes à gérer seuls l'obstacle de l'acquisition foncière. A l'inverse de la situation britannique, ce ne sont donc pas les mêmes opérateurs qui développent les opérations foncières de viabilisation et les opérations immobilières de construction. La spécificité belge de l'auto-promotion est également intimement liée au caractère peu concentré du secteur de la construction résidentielle (0.Dubois, 2001, p. 85). Pour le segment de l'habitat unifamilial, la Belgique se caractérise par quelques entreprises moyennes actives dans le domaine du "clé sur porte " et par de nombreux petits entrepreneurs au profil plus artisanal. En général, ces opérateurs se limitent à la phase de construction et laissent les candidatsbâtisseurs gérer l'acquisition foncière, grâce à l'intervention de promoteurs fonciers qui correspondent souvent à des entreprises spécialisées ou, pour les opérations les plus simples, aux propriétaires initiaux.

\section{B. La représentation de la filière " promoteur professionnel » : analyse des disparités spatiales}

Pour la période 1997-2002, ce sont 146530 nouvelles maisons unifamiliales qui ont fait l'objet d'une autorisation administrative en Belgique ${ }^{8}$. La représentation des permis introduits par des particuliers y est de $82,2 \%$, ce qui atteste de la spécificité belge de l'auto-promotion et, parallèlement, en confirme la persistance ${ }^{9}$. Les permis délivrés à l'attention des promoteurs professionnels privés sont bien moins nombreux. Ils ne représentent que $14,3 \%$ du total. Cette statistique indique que, quel que soit le maître d'œuvre en charge de la conception technique (architecte ou entreprise de construction générale), ce sont bien les particuliers qui sont officiellement responsables des démarches administratives. Le solde de 3,5\% correspond aux permis délivrés à l'attention des maîtres d'ouvrage publics.

Nous avons mené différents tests statistiques afin d'analyser les disparités spatiales de la filière $\mathrm{du}$ " promoteur professionnel » en matière de constructions unifamiliales. Sur base des recherches bibliographiques, nous avons vérifié qu'une faible disponibilité en offre et que des prix élevés sont des facteurs susceptibles de contribuer au 
développement de la filière promoteur. Comme pour les traitements statistiques consacrés aux superficies moyennes des lots constructibles, les tests ont également été menés pour les deux niveaux spatiaux de la commune et du bassin d'emploi. Des tests menés pour le niveau communal, il ne ressort aucune variable significative. À cette échelle, nous ne vérifions ni l'influence du prix des parcelles, ni l'influence de la disponibilité foncière. Par contre, agréger les données au niveau des bassins d'emploi conduit à vérifier l'influence de la disponibilité foncière (graphique 5). Cette observation confirme les développements présentés ci-dessus à propos des relations qu'entretiennent les mécanismes de la production foncière et les ventilations internationales entre la filière de "l'auto-promotion " et la filière du "promoteur professionnel ». Là où la planification est peu rigoureuse et le ratio de disponibilité foncière peu élevé, les particuliers sont aptes à lever l'obstacle de l'acquisition foncière et les professionnels de la construction immobilière sont peu tentés par une intervention directe sur les marchés fonciers. Par contre, lorsque le zonage est plus strict et l'offre - à la fois potentielle et effective - plus limitée, les particuliers éprouvent des difficultés pour acquérir un terrain et l'on peut assister au développement de la filière du promoteur professionnel.

Graphique 5 : Part de la filière promoteur en fonction du ratio de disponibilité foncière.

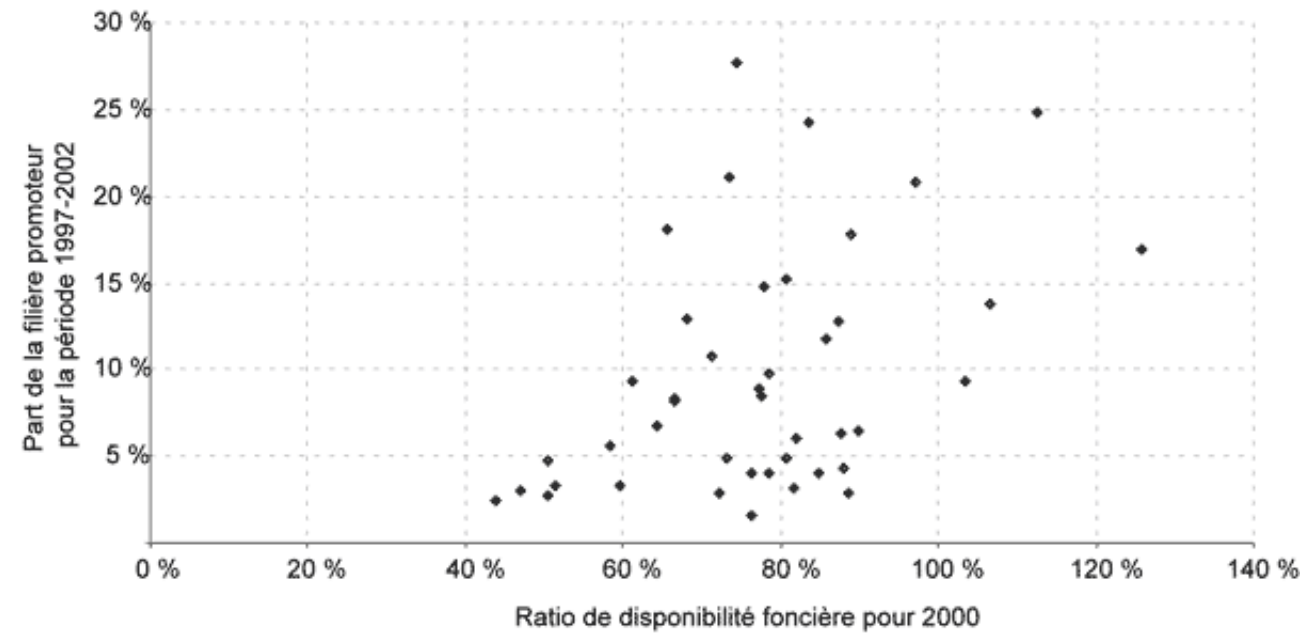

Ensemble de la Belgique à l'échelle des bassins d'emploi (sauf Flandre occidentale et BruxellesCapitale).

Sources: INS (statistiques cadastrales sur l'occupation du sol et statistiques de la construction), DGATLP et ARHOM.

De nos entretiens auprès de promoteurs-constructeurs, il ressort que la maîtrise d'ouvrage plus importante des professionnels au sein des bassins où l'offre est restreinte s'explique principalement par la limitation du risque associé à la commercialisation. En effet, lorsque l'offre foncière est abondante, il est risqué pour un constructeur de se lancer dans la maîtrise d'ouvrage. Dans ce contexte où les particuliers peuvent aisément accéder au marché foncier, mener sa propre opération sera souvent préféré à l'acquisition d'un logement standard déjà réalisé. Bien que neuf, le logement mis en place par un constructeur ne satisfait pas entièrement la demande en raison de la composante symbolique non négligeable d'un produit tel que la maison individuelle. Placement lié au développement de la famille, la maison individuelle neuve est autant recherchée pour sa symbolique et ses attributs d'authenticité que 
pour ses attributs de confort (P. Bourdieu et al., 1990). Il ressort également de nos entretiens auprès de firmes à la fois actives en support à l'auto-promotion et en maitrise d'ouvrage pour leur propre compte que de bonnes perspectives en matière de commercialisation poussent les entreprises du secteur de la construction à assurer leur propre maitrise d'ouvrage. En effet, les constructeurs préfèrent cette alternative car les chantiers peuvent être menés plus efficacement lorsqu'ils sont pleinement responsables des opérations et lorsqu'ils ne doivent pas se soumettre aux sollicitations variées des familles auto-promotrices.

Observer que la relation entre la disponibilité foncière et la représentation de la filière promoteur est significative au niveau des bassins d'emploi mais pas au niveau des communes signifie qu'une pénurie en terrains constructibles à l'échelle communale n'est pas suffisante pour limiter le risque d'une mauvaise commercialisation. En comparaison du niveau communal, le niveau du bassin ressort donc comme une échelle plus appropriée afin d'appréhender l'étendue des territoires prospectés par les ménages candidats-bâtisseurs. Il s'agit là d'une conclusion qui rentre en parfaite cohérence avec nos travaux relatifs à la structure spatiale des marchés fonciers. En effet, différents traitements menés sur cette thématique démontrent que le niveau du bassin est un niveau plus pertinent que le niveau communal pour rendre compte de l'organisation des échanges en terres constructibles (J.-M. Halleux, 2005).

Il ressort de la prise en compte de la littérature que des niveaux fonciers élevés sont, à l'instar d'une faible disponibilité en offre, susceptibles de pousser les promoteursconstructeurs à alimenter le marché avec leurs propres réalisations immobilières, cela en fait afin de bénéficier d'un surprofit (0. Dubois, 2001, p. 43). Dans une telle configuration de marché, les professionnels de la construction peuvent en effet espérer des niveaux de commercialisation élevés et un profit supérieur à la marge de promotion. Cette hypothèse n'a toutefois pas été vérifiée par nos traitements et nous n'observons pas de relations significatives - qu'il s'agisse du niveau communal ou du niveau du bassin - entre les prix fonciers et l'intensité de la filière du promoteur professionnel. Cela résulte de la forte corrélation observée entre les prix fonciers et les niveaux de revenus. En effet, là où les prix sont élevés, la demande potentielle est économiquement favorisée et le promoteur-constructeur qui spéculerait sur son renoncement aux attributs d'authenticité que caractérise l'auto-promotion risquerait d'être confronté à une commercialisation difficile.

\section{Conclusion}

L'objectif de cet article était de lever un coin du voile sur les secteurs opaques de la promotion foncière et immobilière en analysant comment ces secteurs économiques influencent la production des nouveaux espaces résidentiels en Belgique. S'il ne faut surtout pas négliger le rôle des promoteurs sur la constitution des périphéries résidentielles, l'analyse du cas belge démontre toutefois que leurs degrés de liberté sont finalement assez réduits et que leurs choix d'investissements sont très largement déterminés et contraints par la configuration des marchés fonciers, à l'image finalement du degré de liberté des ménages désireux de bénéficier d'une maison unifamiliale neuve. De la même manière qu'il est simpliste de considérer que les choix résidentiels des ménages ne s'expliquent que par leur aspiration pour la maison unifamiliale et le modèle périurbain, il est simpliste de considérer que les choix 
d'investissements des promoteurs s'expliquent uniquement par l'axiome de la maximisation du profit. Pour rendre compte des choix opérés par les différents acteurs actifs en matière de constitution des territoires périurbains, il faut en fait faire référence à de complexes mécanismes systémiques où interfèrent de multiples contraintes, notamment financières pour les ménages, et liées au risque d'une mauvaise commercialisation pour les opérateurs fonciers et immobiliers.

Concernant les promoteurs immobiliers actifs sur le segment de la maison unifamiliale, c'est la localisation des terrains sur lesquels ils vont pouvoir construire qui est déterminée par les caractéristiques des marchés fonciers. En effet, il ressort clairement de nos traitements que les constructeurs ne se risquent pas à alimenter le marché immobilier avec des constructions unifamiliales neuves là où les particuliers peuvent aisément accéder à l'acquisition d'une parcelle et, dès lors, aux caractéristiques d'authenticité associées à la filière de l'auto-promotion. Révélatrice de la dispersion des chantiers et de l'éparpillement périurbain qui caractérise les périphéries belges, la prépondérance de l'auto-promotion qui y est observée est donc liée à la tradition faiblement planificatrice du pays et, en comparaison des contextes britanniques, néerlandais et danois, à l'abondance en terrains juridiquement bâtissables qui accompagne cette tradition. De ce point de vue, la périurbanisation particulièrement éclatée qui affecte la Belgique paraît donc s'expliquer par la conjonction d'une société qui, comme ses voisines atlantiques, aspire très profondément à l'isolement unifamilial, mais qui, à la différence de ses voisines nordiques, ne dispose pas de la capacité planificatrice suffisante afin d'éviter que cet isolement se disperse dans la ressource foncière.

A l'instar de leurs collègues actifs en promotion immobilière, les promoteurs actifs dans le lotissement sont également très fortement contraints par le risque d'une mauvaise commercialisation. Il s'agit là d'un constat qui permet d'expliquer pourquoi la périurbanisation belge s'accompagne d'une consommation d'espace extrêmement importante. En effet, il apparaît clairement qu'une meilleure rentabilisation des gisements par la réduction de la superficie des parcelles constructibles n'est praticable qu'en situation de hauts niveaux de prix et de faible disponibilité. Par contre, lorsque le marché des lots n'est que faiblement sous pression, le lotisseur qui prendrait le risque de resserrer ses parcelles en vue d'accroître son profit serait incapable d'attirer la demande si des concurrents moins téméraires choisissent d'alimenter le marché avec de grandes superficies.

\section{BIBLIOGRAPHIE}

BALL M. (2003). - « Markets and the Structure of the Housebuilding Industry : An International Perspective ", Urban Studies, Vol. 40, n 5/6, p. 897-916.

BOURDIEU P., (avec la collaboration de) BOUHEDJA S., CHRISTIN R. et GIVRY C. (1990). - « Un placement de bon père de famille. La maison individuelle : spécificité du produit et logique du champ de production ", Actes de la recherche en sciences sociales, $\mathrm{n}^{\circ}$ 81-82, p. 6-33. 
BRAMley G., BARTLETT W. et LAMBERT C. (1995). - Planning, the Market and Private Housebuilding, The natural and built environnment series 4, London University College Press, Londres.

Brück L., Halleux J.-M., Jehin J.-B., Lambotte J.-M., Mairy N., Van Hoof T., SAVenberg S., sous la direction de Mérenne-Schoumaker B. et Van Hecke E. (2000). - Summary of the project « Residential Behaviour of Household and Sustainable Development Issue ", SSTC - Leviers d'une politique de développement durable, Université de Liège, SEGEFA-ISEG, Université de Liège - KULeuven, inédit. Disponible sur : http://www.ulg.ac.be/geoeco/segefa/

Colwell P.F. et MunNeKe H.J. (1997). - « The Structure of Urban Land Prices », Journal of Urban Economics, $\mathrm{n}^{\circ} 41$, p. 321-336.

Cомву J. (2003). - « La formation de la valeur sur les six marchés fonciers », Etudes foncières, $\mathrm{n}^{\circ} 101$, p. 18-23.

COMBY J., RENARD V. (1996). - Les politiques foncières, Coll. « Que sais-je ? », PUF, Paris.

De la Morvonnais P. (1998). - «Comparaisons internationales », Logement et habitat l'état des savoirs (sous la direction de Segaud M., Bonvalet C. et Brun J.), La Découverte, Paris, p. 147-157.

De WAsseige Y., LAFfut M., RuYTers C., SCHeilPer P. (2000). — « Bassins d'emploi et régions fonctionnelles. Méthodologie et définitions des bassins d'emploi belges ", Discussion Papers, $\mathrm{n}^{\circ}$ 2005, Service des Etudes et de la Statistique, Ministère de la Région wallonne.

Dubois O. (2001). - La construction résidentielle en Wallonie : analyse spatiale multiscalaire et logiques socio-économiques de localisation, Thèse présentée en vue de l'obtention du grade de docteur en environnement, IGEAT, ULB, inédit.

Duвois O. (2002). - « Stratégie des acteurs de la construction résidentielle neuve et caractéristiques des espaces bâtis en Belgique », BELGEO, ${ }^{\circ} 4$, p. 319-332.

Dubois O., Gabriel I., HalleuX J.-M., Michel Q. (2002). - Révision des plans de secteur et mécanismes fonciers en Wallonie : objectifs politiques, outils juridiques et mise en œuvre, Etudes et Documents CPDT 2, Ministère de Région wallonne, DGATLP, Namur.

Dubois O. et HALlEUX J.-M. (2003). - « Marchés immobiliers résidentiels et étalement périurbain contraint. L'accessibilité au logement au sein des communes wallonnes de la région métropolitaine bruxelloise », BELGEO, $\mathrm{n}^{\circ}$ 3, p. 303-327.

DunCAN S.S., RowE A. (1993). - « Self-provided Housing : “The first World's Hidden Housing Arm" ", Urban Studies, Vol. 30, n 8, p. 1331-1354.

FOUCHIER V. (2001). - « Mesurer l'étalement, la dédensification, le desserrement : différentes formes de gain d'espaces en Ile-de-France ", La ville aux champs (ADEF), Paris, p. 29-48.

HALlEuX J.-M. (2002a). - « Outskirts of European cities : Commented bibliography on Belgium », Outskirts of European cities - state of the art report, COST Action C10, European Commission, Brussels, p. 91-112. Disponible sur : http ://www.qub.ac.uk/ep/research/costc10/stateofart.pdf HALLEUX J.-M. (2002b). - « Le territoire wallon et sa surabondance foncière : pourquoi et comment gérer l'offre de manière parcimonieuse ", Actes des 2es Rencontres de la Conférence Permanente du développement Territorial, Ministère de la Région wallonne, CPDT, Jambes, p. 21-30.

HALlEUX J.-M. (2005). - Structuration spatiale des marchés fonciers et production de l'urbanisation morphologique. Application à la Belgique et à ses nouveaux espaces résidentiels, Etude de géographie économique, Dissertation présentée le 24 juin 2005 en vue de l'obtention du grade de Docteur en Sciences, Université de Liège, inédit. 
HALLEUX J.-M., BRÜCK L., MAIRY N. (2002). - « La périurbanisation résidentielle en Belgique à la lumière des contextes suisses et danois : enracinement, dynamiques centrifuges et régulations collectives ", BELGEO, n 4, p. 333-354.

Halleux J.-M., Lambotte J.-M., Kessler L., Pirotte B., Sluze M. (2003). - « Les blocages du recyclage morphologique des tissus urbains ", Reconstruire la ville sur la ville, Recyclage des espaces dégradés, Ministère de la Région wallonne, CPDT, inédit, p. 33-115.

JEHIN J.-B. (1998). - « La périurbanisation et la rurbanisation à travers la consommation d'espace ", Bulletin de la Société géographique de Liège, Vol. 34, p. 45-52.

LÉVY J. (1997). - L’Europe. Une géographie, Hachette, Paris.

Kesteloot C., De Maesschalck S. (2001). - « Anti-urbanisme in Flanders : the political and social consequences of a spatial class struggle strategy », BELGEO, n 1-2, p. 41-62.

Topalov C. (1974). - Les promoteurs immobiliers. Essai d'analyse d'un système d'acteurs, Economica, Paris.

ViNCENT M. (1987). - « Filières de production du logement et modes de formation des prix ", Espaces et sociétés, $\mathrm{n}^{\circ}$ 51, p. 59-81.

VAn der Haegen H., VAn HeCKe E., Juchtmans G., 1998, « Les régions urbaines », Monographie n 11A «Urbanisation » du Recensement Général de la Population et des Logements au 1er mars 1991 (sous la direction de Mérenne-Schoumaker B., Van der Haegen H. et Van Hecke E.), INS, SSTC, Bruxelles, p. 79-148.

\section{NOTES}

1. Le lecteur intéressé pourra faire référence au bilan bibliographique présenté par J.M. Halleux (2002a) dans le cadre de l'action Cost C10.

2. Cette situation tranche en comparaison du contexte français où les zones dédiées à l'urbanisation résidentielle font l'objet de révisions fréquentes.

3. À propos de l'élaboration du ratio de disponibilité foncière, remarquons que la variable n'est pas satisfaisante pour la province de Flandre occidentale. Cette province se caractérisant par un important habitat dispersé et par de nombreuses habitations situées en dehors des zones d'habitat, la méthode employée pour apprécier l'offre juridique demeurant vierge s'y est malheureusement révélée inappropriée, ce qui nous a dès lors contraint à ne pas considérer cette partie du territoire.

4. Le découpage du pays en bassins d'emploi a été réalisé par le SES (service d'études et de statistiques) de la Région wallonne (Y. De Wasseige et al., 2000). Cette délimitation présente l'avantage d'avoir été établie de manière homogène sur l'ensemble du Royaume, à partir des données collectées lors du recensement INS de 1991 sur les migrations pendulaires de travail. La méthodologie utilisée par les chercheurs du SES est celle de la classification hiérarchique ascendante. Elle consiste dans la constitution d'un arbre organisant, de manière hiérarchisée, les relations entre les 589 communes du pays. Le traitement a conduit à sélectionner 46 bassins d'emploi relativement indépendants les uns des autres en matière de navettes de travail.

5. Cette typologie prend en compte les 17 complexes résidentiels urbains (CRU) du Royaume en différenciant cinq groupes annulaires:- les villes centrales, soit les municipalités correspondant aux centres-villes traditionnels;- les communes d'agglomération, c'est-à-dire les entités marquées par une urbanisation morphologique continue depuis la ville centrale ; - la banlieue périurbaine, formée des communes dont la croissance démographique des dernières 
décennies a été alimentée par le déversement en provenance de l'agglomération et de la ville centrale ; - la zone résidentielle des migrants alternants (ZRMA), rattachée au CRU en raison d'un fort développement de navettes pendulaires autochtones vers l'agglomération et la ville centrale ;- la catégorie hors CRU, formée des 214 municipalités situées au-delà des zones résidentielles des migrants alternants.Pour le lecteur peu familiarisé avec la terminologie relative au phénomène des régions urbaines, précisons encore que la "région urbaine » correspond à l'ensemble formé par la commune centrale, l'agglomération et la banlieue périurbaine. L'expression "complexe résidentiel urbain" fait pour sa part référence à un territoire plus vaste qui, en plus des trois premiers groupes, intègre également la ZRMA.

6. Comme nous le verrons ci-dessous, la prise en compte de données récentes confirme la persistance de l'auto-promotion observée en Belgique en matière de nouveaux développements résidentiels.

7. La formule « candidat-bâtisseur » est fréquemment utilisée en Belgique. L'usage courant de cette expression, qui s'applique à des particuliers, illustre la prédominance de l'auto-promotion dans la constitution des stocks de logements.

8. Source : Institut National de Statistiques, Statistiques de la construction.

9. Nous ne disposons malheureusement pas de données antérieures à 1997 afin de réaliser une analyse évolutive.

\section{RÉSUMÉS}

Malgré l'importance des enjeux spatiaux et économiques qu'ils représentent, les secteurs de la promotion foncière et immobilière demeurent mal connus. L'objectif de cet article, consacré au contexte spatial des nouvelles urbanisations résidentielles produites en Belgique, est d'analyser le rôle de ces secteurs sur l'utilisation du sol urbain et sur la production des périphéries. En première partie, nous présentons le contexte spatial étudié. En deuxième partie, consacrée aux choix d'investissement des promoteurs fonciers, nous nous efforçons de comprendre pourquoi la périurbanisation belge s'accompagne d'une consommation d'espace extrêmement importante. En troisième partie, la prise en compte de la promotion immobilière permet d'expliquer la particularité belge de la forte prédominance de l'auto-promotion dans la constitution des stocks de logements. Au final, notre analyse démontre que les choix d'investissements des promoteurs fonciers et immobiliers sont très fortement contraints par la configuration des marchés fonciers.

Despite their spatial and economic importances, the sectors of property and land developments are still badly known. The aim of this article, dedicated to the spatial context of new residential developments produced in Belgium, is to analyse the influence of those sectors on both, the urban land-uses and the characteristics of residential outskirts. In the first part, the analysed spatial context is presented. In the second part, dedicated to the investment choices of land developers, we try to apprehend why the Belgian peri-urbanisation engenders very important land consumptions. In the third part, the property development issue is taken into account in order to explain why the self-provided sector is so highly prevailing in Belgium. Our research leads to the conclusion that investment choices of both, land developers and property developers, are highly constrained by land market configurations. 
Trotz ihrer räumlichen und ökonomischen Bedeutung sind die Eigentumsverhältnisse und die Tätigkeiten von Wohnungsbaugesellschaften bislang nur unzureichend wissenschaftlich untersucht worden. Der vorliegende Artikel zielt darauf ab, diese für die Stadtentwicklung so wichtigen Sektoren zu beleuchten und die neuen Wohnviertel, die in Belgien entstanden sind, unter diesem Gesichtspunkt zu untersuchen. Die Analyse der Eigentumsstrukturen und der Stadtentwicklung ermöglicht ein besseres Verständnis der Akteure des Wohnungsbaus und der entstandenen Strukturen der städtischen Außenviertel. Es zeigt sich, dass die Charakteristiken des Bodenmarktes ist hohem Maße die Handlungsspielräume der Akteure determinieren.

\section{INDEX}

Schlüsselwörter : Belgien, Bodenmarkt, Suburbanisierung, Wohnbaugesellschaft Keywords : Belgium, land development, land market, peri-urbanisation, property development Mots-clés : Belgique, marché foncier, périurbanisation, promotion foncière, promotion immobilière

\section{AUTEUR}

\section{JEAN-MARIE HALLEUX}

Université de Liège, Unité de géographie économique et sociale, Place du 20 Août, 4000 Liège, Belgique 